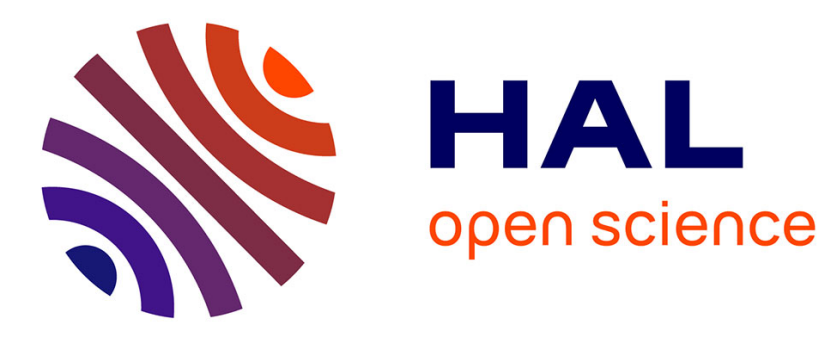

\title{
(Not so) Intuitive Results from a Smart Agriculture Low-Power Wireless Mesh Deployment
}

Keoma Brun-Laguna, Ana Laura Diedrichs, Diego Dujovne, Rémy Leone, Xavier Vilajosana, Thomas Watteyne

\section{- To cite this version:}

Keoma Brun-Laguna, Ana Laura Diedrichs, Diego Dujovne, Rémy Leone, Xavier Vilajosana, et al.. (Not so) Intuitive Results from a Smart Agriculture Low-Power Wireless Mesh Deployment. CHANTS'16, Sep 2016, New York City, United States. 10.1145/2979683.2979696 . hal-01361333

\section{HAL Id: hal-01361333 \\ https://inria.hal.science/hal-01361333}

Submitted on 7 Sep 2016

HAL is a multi-disciplinary open access archive for the deposit and dissemination of scientific research documents, whether they are published or not. The documents may come from teaching and research institutions in France or abroad, or from public or private research centers.
L'archive ouverte pluridisciplinaire HAL, est destinée au dépôt et à la diffusion de documents scientifiques de niveau recherche, publiés ou non, émanant des établissements d'enseignement et de recherche français ou étrangers, des laboratoires publics ou privés. 


\title{
(Not so) Intuitive Results from a Smart Agriculture Low-Power Wireless Mesh Deployment
}

\author{
Keoma Brun-Laguna \\ Inria, EVA team, \\ Paris, France \\ keoma.brun@inria.fr \\ Ana Laura Diedrichs \\ Universidad Tecnológica Nacional (UTN) \\ Mendoza, Argentina \\ ana.diedrichs@frm.utn.edu.ar \\ Diego Dujovne \\ Universidad Diego Portales \\ Santiago, Chile \\ diego.dujovne@mail.udp.cl \\ Xavier Vilajosana \\ Univ. Oberta de Catalunya, \\ Barcelona, Catalonia, Spain \\ xvilajosana@uoc.edu \\ Rémy Léone \\ Inria, EVA team, \\ Paris, France \\ remy.leone@inria.fr \\ Thomas Watteyne \\ Inria, EVA team, \\ Paris, France \\ thomas.watteyne@inria.fr
}

September 7, 2016

\begin{abstract}
A 21-node low-power wireless mesh network is deployed in a peach orchard. The network serves as a frost event prediction system. On top of sensor values, devices also report network statistics. In 3 months of operations, the network has produced over 4 million temperature values, and over 350,000 network statistics. This paper presents an in-depth analysis of the statistics, in order to precisely understand the performance of the network. Nodes in the network exhibit an expected lifetime between 4 and 16 years, with an end-to-end reliability of $100 \%$. We show how contrary to popular belief - wireless links are symmetric. Thanks to the use of Time Slotted Channel Hopping (TSCH), the network topology is very stable, with $\leq 5$ link changes per day in the entire network.
\end{abstract}




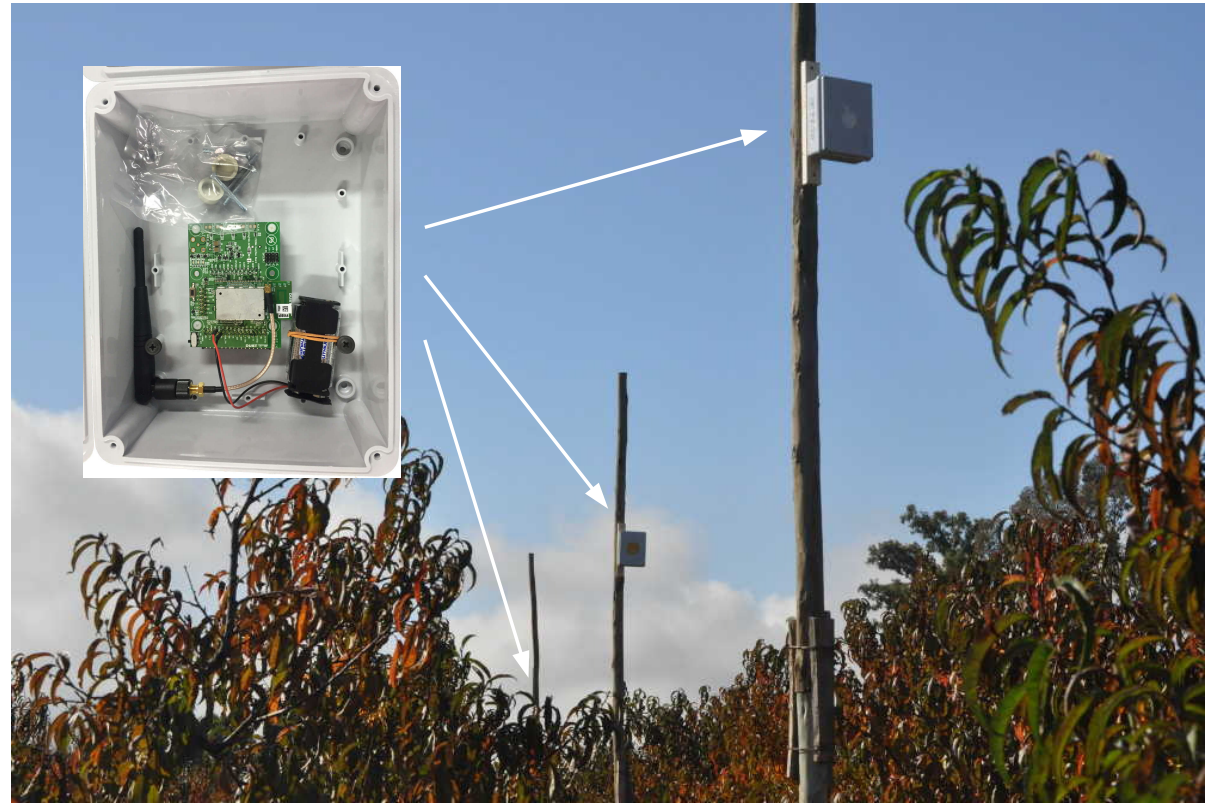

Figure 1: The wireless motes deployed in the peach orchard in Mendoza, Argentina.

\section{Introduction}

Peaches don't like frost. If during the blooming season (September in Argentina), temperature gets below $-3 \mathrm{C}$ for only a couple of hours, the flowers freeze, and no peaches are produced. In $2013,85 \%$ of the peach production in the Mendoza region (western Argentina) was lost because of frost events. Farmers can lose everything in only a couple of hours. Yet, if they are warned of a frost event a couple of hours ahead, they can install heaters throughout the orchards, and use big fans to move the hot air around. Fighting the frost events is not the issue, what is hard is predicting it.

The goal of the PEACH project [7] is to predict frost events. We install sensors around the orchard that measure air temperature, air relative humidity, soil moisture and soil temperature. We feed the collected data into a database, and by analyzing the data in real-time using machine learning, we identify patterns in the data and predict frost events.

Because of the heavy machinery that moves inside the orchard, using cables to interconnect the sensors is not an option. The main challenge is to deploy a system that provides both a high end-to-end reliability and a long lifetime without using cables. We use SmartMesh IP, an off-the-shelf low-power wireless mesh solution from Linear Technology. The sensor devices are battery-powered and equipped with a radio. They form a multi-hop topology, and collaborate to route the data generated by the devices (called "motes") to a gateway. This 


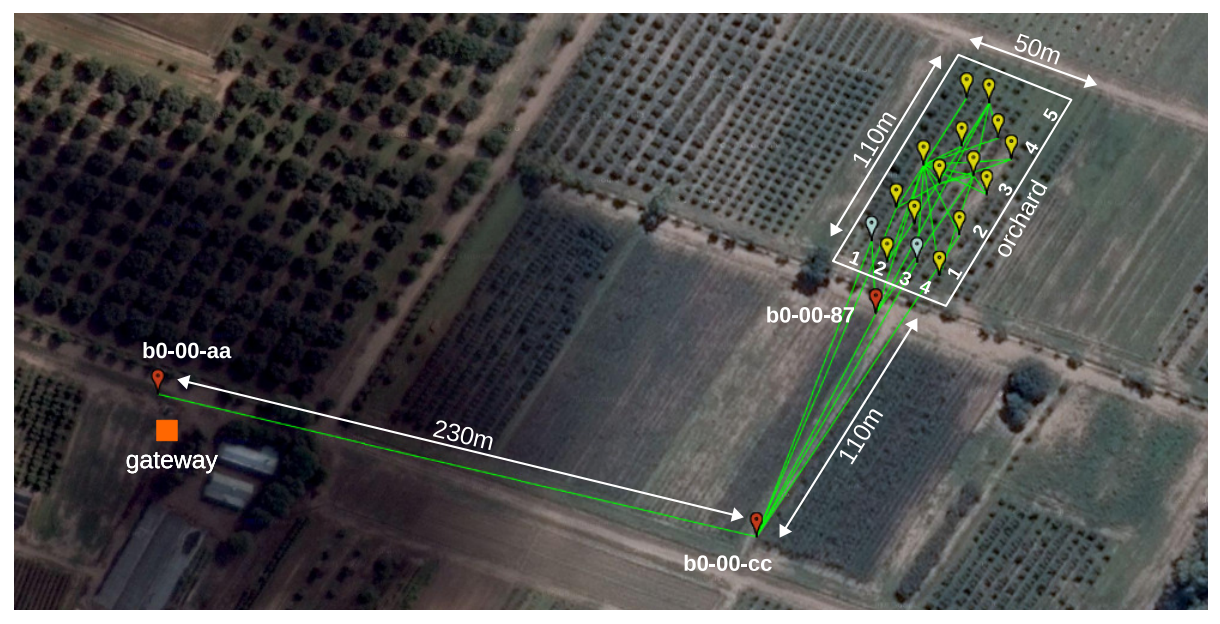

Figure 2: Areal view of the sensor network deployed in the orchard near Mendoza, Argentina.

gateway is connected to the Internet, and forwards the data gathered in the peach orchard in Argentina to the PEACH servers in Paris, France. Data appears on the web interface of the servers seconds after it was gathered in the orchard.

The network is deployed in a peach orchard of 204 trees, planted in a $50 \mathrm{~m} \times 100 \mathrm{~m}$ area (shown in Fig. 2). The low-power wireless network is composed of 18 sensor motes ${ }^{1}$ uniformly distributed between the peach trees, and 3 relay motes to connect the orchard to the gateway some $300 \mathrm{~m}$ away. Each mote is placed in a water-tight box that is fixed on a $4 \mathrm{~m}$ high pole (see Fig. 1).

\footnotetext{
${ }^{1} 2$ motes malfunctioned and are not present on the map.
} 


\begin{tabular}{|c|c|c|c|c|}
\hline $38-0 f-66$ & $60-05-78$ & & & 5 \\
& $60-05-a b$ & $60-02-4 b$ & $60-02-1 b$ & 4 \\
$60-05-5 f$ & $60-06-27$ & $60-05-69$ & $60-01-f 8$ & 3 \\
$3 f-f e-87$ & $3 f-f e-88$ & & $60-08-d 5$ & 2 \\
$30-60-$ f $^{*}$ & $3 f-f 8-20$ & $58-32-36^{*}$ & $60-03-82$ & 1 \\
\hline 1 & 2 & 3 & 4 & \\
\hline
\end{tabular}

Table 1: The MAC addresses of the motes inside the orchard (last 3 bytes). Refer to numbers displayed on the map. (*) DC9018 node with external antenna.

We use four types of SmartMesh IP devices. The 2 DC9018 boards feature an external antenna; the 16 DC9003 boards a chip antenna. These are deployed inside the orchard. We deploy 3 repeaters outside the orchards to connect the orchard to the gateway. The gateway is composed of a Raspberry Pi singleboard computer, and a DC2274 SmartMesh IP manager.

The SmartMesh IP network implements the IEEE802.15.4e standard [2], which includes a channel hopping mechanism to reduce the impact of multipath fading and external interference. This allows the network to be highly reliable, stable, and extremely low power $[8,9]$.

Each mote produces a temperature value every $30 \mathrm{~s}$, and network statistics every $5 \mathrm{~min}$. In 3 months of operation, we gathered over 4 million temperature values, and more than 350,000 network statistics.

The goal of this paper is to analyze the network statistics over a 3-month period, and precisely assess the performance of the network. 
This paper makes the following contributions:

- We confirm that the SmartMesh IP network exhibits years of battery lifetime and wire-like reliability;

- We show that channel hopping causes the network topology to be very stable, with $\leq 5$ link changes per day;

- Contrary to popular belief, we show that links in the network are symmetric, i.e. they exhibit the same signal strength in both directions of the same link.

The remainder of this paper is organized as follows. Section 2 describes what statistics we are collecting, and the amount of statistics collected over a 3 month period. Section 3 presents results that confirm assumptions about what we can expect for real-world SmartMesh IP deployment. Section 4 presents not so intuitive results about link symmetry and network stability. Finally, Section 5 concludes this paper and discusses further improvements.

\section{Statistics Collected}

The wireless network is deployed in a peach orchard in Junin, $45 \mathrm{~km}$ South-East of Mendoza in Western Argentina. No other electronic devices are present in the field. Farmers work inside the field with heavy machinery for 1-2 h every 20 days approximately. In the region, air temperature ranges between $-9 \mathrm{C}$ in winter (May-October) to $+38 \mathrm{C}$ in summer (November-April). Because of the sunny weather, day/night temperature swings of $10+\mathrm{C}$ are not uncommon in winter.

Each device in the network produces both sensor data and network statistics. Network statistics can be separated in Events and Health Reports messages. Event messages are non-periodic notifications the network sends when a network event happens (e.g. a node joins/leaves the network, a link is created/deleted). Health Report (HR) messages are sent periodically by each mote; they contain counters and statistics about that mote. HRs are used to assess the overall health of the network.

Table 2 summarizes the number of events and HRs gathered during the 3 month period. In the remainder of this section, we detail the meaning of each of the statistics.

mote_create. Each node in a SmartMesh IP network can periodically send beacons to announce the presence of the network. When a mote wants to join a network, it listens for those beacons. Once it has heard a number of those, it starts a security handshake with the network. During that handshake, the SmartMesh IP manager sends a mote_create event notification over its serial port. This is the event we $\log ^{2}$. It contains, among other information, the

\footnotetext{
${ }^{2}$ Normally, each mote generates a single mote_create event. Due to power issues at the manager side, the network restarted a couple of times and new events were created.
} 


\begin{tabular}{|l|r|}
\hline \multicolumn{1}{|c|}{ type } & number \\
\hline \hline mote_create & 133 \\
\hline path_create & 4,098 \\
\hline path_delete & 3,653 \\
\hline HR_DEVICE & 132,758 \\
\hline HR_DISCOVERED & 87,737 \\
\hline HR_NEIGHBORS & 140,897 \\
\hline
\end{tabular}

Table 2: The number of statistics collected over the 3 month period.

association between the newly-joined device's 8-byte MAC address and its 2byte moteId.

path_create and path_delete. In SmartMesh IP terminology, a "path" is the link-layer resource that allows two neighbor nodes to communicate ${ }^{3}$. Each time a mote starts communicating with a new neighbor (e.g. its routing parent), a path_create event is produced. Similarly, each time a mote stops communicating with a neighbor (e.g. it changes routing parent), a path_delete event is produced. We log both messages.

HR_DEVICE. Each network device produces a HR_DEVICE every 15 min. This health report contains counters/statistics internal to the mote, such as its current battery voltage, temperature, or total number of messages sent.

HR_DISCOVERED. SmartMesh IP nodes continuously monitor their surroundings to discover neighbor nodes. Every 15 min, each node produces an HR_DISCOVERED health report that contains the list of "discovered" neighbors, and the associate signal strength it heard them at. These discovered neighbors can potentially be used in the future as neighbors the node communicates with.

HR_NEIGHBORS. Two nodes are neighbors when link-layer resources are installed for them to communicate. The neighbors of a node are a subset of the discovered neighbors. Every 15 min, each note generates an HR_NEIGHBORS health report that contains its list of neighbors. These messages also specifies per-neighbor counters, such as the number of link-layer retransmissions.

After 3 months of operation, we have collected 369,276 network statistics (see Table 2). The goal of the next section is to present the main results from analyzing this information. We group these results in two categories. "Intuitive" results (Section 3) are results that confirm the performance expected from a SmartMesh IP network. "Not so intuitive" results (Section 4) are results that we believe go against popular belief. This classification is necessarily subjective.

Possibly due to power line failure at the network manager side, the network experienced some restarting. For this reason, some analysis presented in the next sections are done in shorter period. As a side effect, this allows us to verify the network formation and joining process.

\footnotetext{
${ }^{3}$ In more classical networking terminology, this is often referred to as a "link". We use the terms "path" and "link" interchangeably in this paper.
} 


\section{Intuitive Results}

Previous publications $[7,8,9,10]$ underline the performance of TSCH networks in general, and SmartMesh IP in particular. Standardization work in the IETF $6 \mathrm{TiSCH}$ working group ${ }^{4}$ around TSCH networks further illustrates the move of the industry towards this type of networking technology. So while we expect good performance from the network, this section verifies that this is indeed the case. We start by looking at two physical-layer metrics: RSSI vs Distance (Section 3.1) and PDR vs. RSSI (Section 3.2). While these have no dependency on TSCH (the type of medium access), they allow us to verify the overall connectivity in the network. We then look at key performance indicators of SmartMesh IP networks: end-to-end reliability (Section 3.3) and network lifetime (Section 3.4).

\subsection{RSSI vs. Distance}

The Friis transmission model [5] gives the relationship between the Received Signal Strength $(\mathrm{RSSI})^{5}$ in free space. While it does not apply directly to our Smart Agriculture outdoor deployment, we note in Fig. 3 that the individual RSSI values are located between the Friis model, and the Friis model offset by $-40 \mathrm{~dB}$. This corroborates the results from [11].

\subsection{Wireless Waterfall}

Due to the inherent physical unreliability of the radio medium, it is impossible to know if a future transmission will be successful or not. The Packet Delivery Ratio (PDR) is the portion of successful link-layer transmissions over the total number of link-layer transmission attempts. A failed attempt means that the link-layer frame needs to be re-transmitted; it does not mean the packet is lost. Over a period of 3 months, 140,897 HR_NEIGHBORS messages are collected. These contain, for a given node, the number of link-layer transmission attempts and successes to each of its neighbors. We remove the portion of neighbors with no transmission and keep only the DC9003 motes, resulting in a total of 88,284 messages (approx. 37\% from the total number of HR_NEIGHBORS).

Fig. 4 plots the PDR and the RSSI of these 125,103 messages. For readability, we also plot the average/deviation of the data for a given RSSI value. Because of its shape, this is known as the "waterfall plot".

Overall, above $-85 \mathrm{dBm}$, the PDR of the link is very good ( $₫ 95 \%)$. Below that value, the PDR rapidly degrades, indicating that, on these links, frequent retransmissions happen. The device manufacturer documentation [4] indicates that a path is considered as "bad" when:

- $\mathrm{RSSI}_{i}-80 \mathrm{dBm}$ and PDR $50 \%$

\footnotetext{
${ }^{4}$ https://tools.ietf.org/wg/6tisch/charters

${ }^{5}$ Strictly speaking, the RSSI is the Received Signal Strength Indicator, a value returned by radio chip. Because of its prevalence in low-power wireless literature, we use it RSS and RSSI interchangeably.
} 


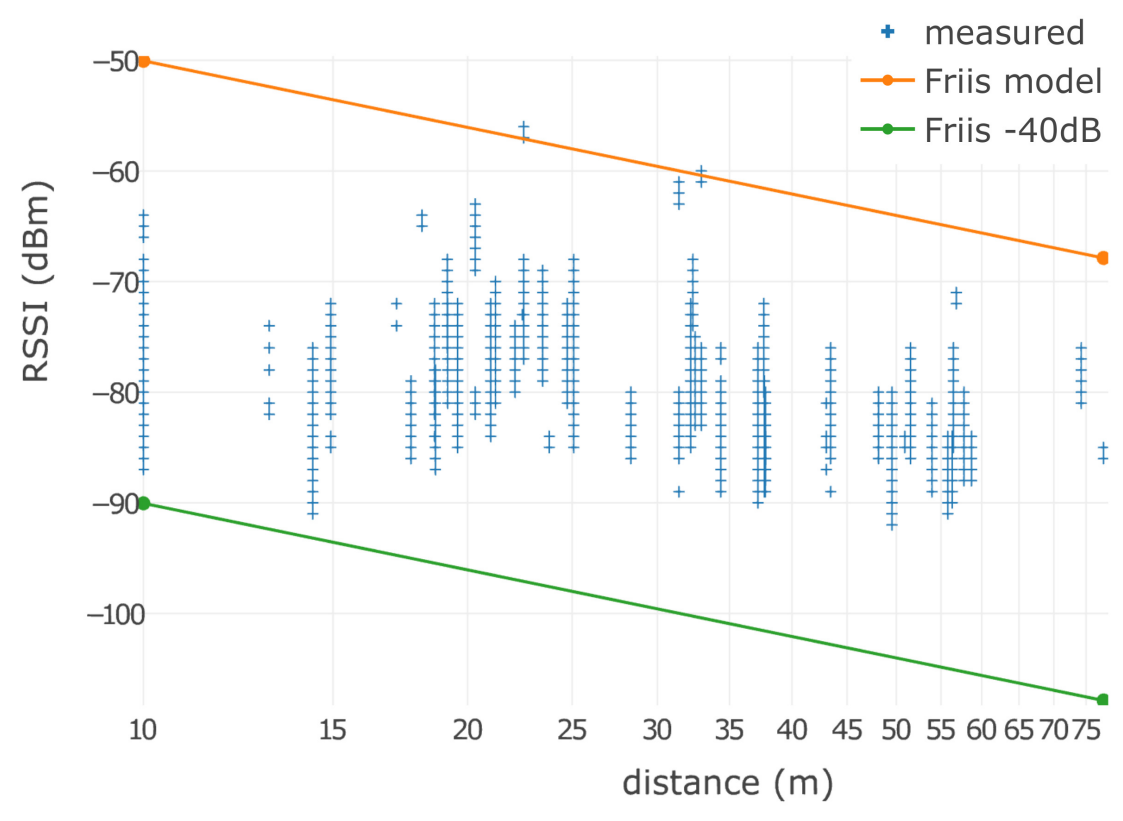

Figure 3: RSSI measurements are roughly located between the Friis model and the Friis model shifted by $-40 \mathrm{~dB}$.

- $\operatorname{RSSI}_{i}-70 \mathrm{dBm}$ and PDR $\mathrm{PD} 70 \%$

This is not the case here.

A waterfall plot either shifted right with very few paths below $-70 \mathrm{dBm}$, or with a non-constantly decreasing curve would be an example of interferenceprone environment. This is not the case in Fig. 4, meaning that the SmartMesh IP network is not experiencing high levels of interferences from co-located wireless devices.

\subsection{End-to-End Reliability}

We expect the SmartMesh IP network to offer wire-like reliability. Table 3 confirms that this is the case. It presents statistics gathered over July 15-25 2016 period.

It shows that, as none of the 693,844 packets generated in the network was lost, the end-to-end reliability is $100 \%$. The average PDR over all the links is very high (95\%), indicating that the nodes are deployed close enough to one another. Finally, the average latency over all nodes is $700 \mathrm{~ms}$. These results are very similar to the very initial results presented in [7], indicating no degradation 


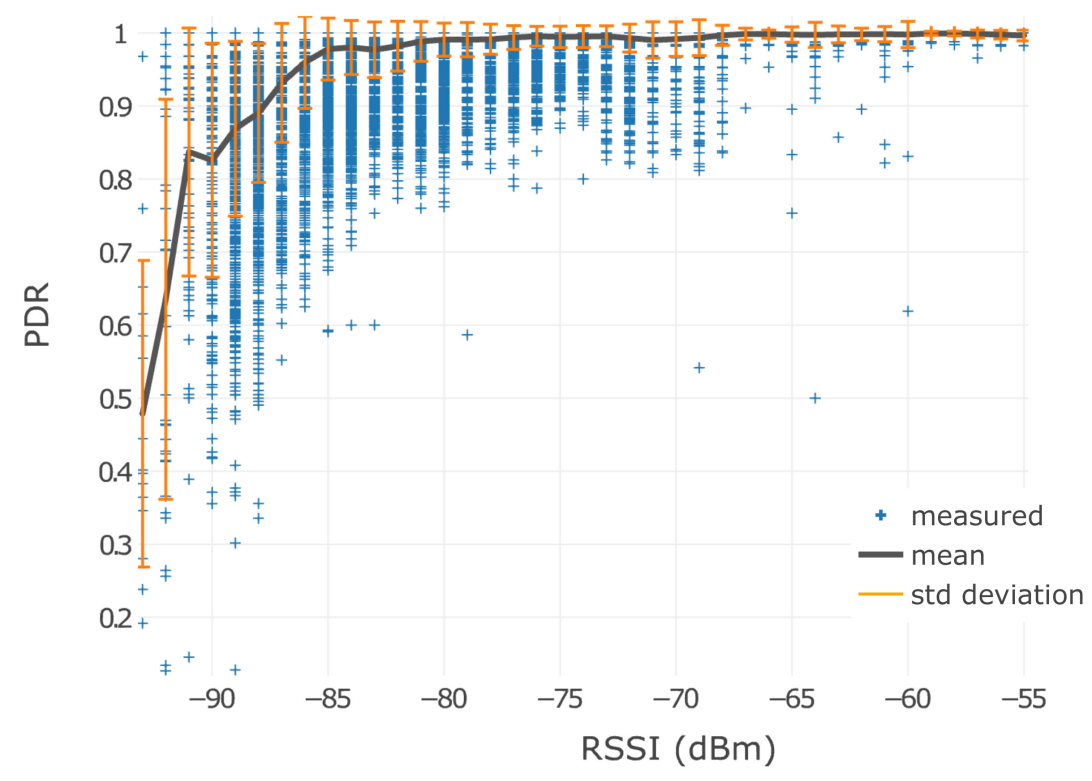

Figure 4: The PDR/RSSI "waterfall" plot.

\begin{tabular}{|l|l|}
\hline reliability & $100 \%$ (Arrived/Lost: $693844 / 0)$ \\
\hline average PDR & $95 \%$ (Transmit/Fails: 4405569/258778) \\
\hline latency & $700 \mathrm{msec}$ \\
\hline
\end{tabular}

Table 3: The overall network performance in the 15-25 July 2016 period. 


\begin{tabular}{|c|c|r|}
\hline MAC address & charge consumed & lifetime \\
\hline $30-60-\mathrm{ef}$ & $227,847 \mathrm{C}(2.2 \%$ battery $)$ & 10.8 years \\
$38-0 \mathrm{f}-66$ & $252,356 \mathrm{C}(2.5 \%$ battery $)$ & 9.8 years \\
$3 \mathrm{f}-\mathrm{f} 8-20$ & $291,312 \mathrm{C}(2.9 \%$ battery $)$ & 8.4 years \\
$3 \mathrm{f}-\mathrm{fe}-87$ & $392,606 \mathrm{C}(3.9 \%$ battery $)$ & 6.3 years \\
$3 \mathrm{f}-\mathrm{fe}-88$ & $458,459 \mathrm{C}(4.5 \%$ battery $)$ & 5.3 years \\
$58-32-36$ & $327,634 \mathrm{C}(3.2 \%$ battery $)$ & 7.5 years \\
$60-01-\mathrm{f} 8$ & $252,454 \mathrm{C}(2.5 \%$ battery $)$ & 9.8 years \\
$60-02-1 \mathrm{~b}$ & $222,253 \mathrm{C}(2.2 \%$ battery $)$ & 10.1 years \\
$60-02-4 \mathrm{~b}$ & $146,068 \mathrm{C}(1.4 \%$ battery $)$ & 16.8 years \\
$60-03-82$ & $494,841 \mathrm{C}(4.9 \%$ battery $)$ & 5.0 years \\
$60-05-5 \mathrm{f}$ & $274,502 \mathrm{C}(2.7 \%$ battery $)$ & 9.0 years \\
$60-05-69$ & $437,136 \mathrm{C}(4.3 \%$ battery $)$ & 5.7 years \\
$60-05-78$ & $304,145 \mathrm{C}(3.0 \%$ battery $)$ & 8.1 years \\
$60-05-\mathrm{ab}$ & $284,764 \mathrm{C}(2.8 \%$ battery $)$ & 8.7 years \\
$60-06-27$ & $321,879 \mathrm{C}(3.2 \%$ battery $)$ & 7.7 years \\
$60-08-\mathrm{d} 5$ & $263,120 \mathrm{C}(2.6 \%$ battery $)$ & 9.3 years \\
\hline
\end{tabular}

Table 4: Per-node power consumption and associated expected lifetime when powered by a pair of AA batteries.

in performance of the SmartMesh IP network over the 3 month operation.

\subsection{Network Lifetime}

Each device is powered by a pair of Energizer L-91 AA batteries. These contain a nominal $3134 \mathrm{mAh}$ of charge, or $2821 \mathrm{mAh}$ when accounting for a $10 \%$ decrease due to manufacturing differences. A SmartMesh IP node contains a "charge accounting" feature in which it tracks the amount of charge is has been drawing from the battery. The mote reports this number every $15 \mathrm{~min}$ as a field in its HR_DEVICE health report. This number allows us to predict the lifetime of the device.

Table 4 shows charge consumed by the 16 motes inside the orchard over the 3 month period (87 days), as well as the portion of the battery this represents. Assuming the same energy consumption rate, we can extrapolate the lifetime. The node with the longest lifetime is $60-02-4 \mathrm{~b}$. From Fig. 2, we can see that this is a leaf node. Since it does not have to relay data from any children, it is normal that this node consumes very little. The node with the shortest lifetime is $60-03-82$ and has 5 years of lifetime. This shows the ultra-low power consumption of the SmartMesh IP network.

\section{Not so Intuitive Results}

Results from Section 3 are "intuitive" is that they corroborate previous measurements $[7]$ or confirm theoretical/lab results $[8,9,10]$. This section presents 
results which we believe go against popular belief. This classification is necessarily subjective.

In Section 4.1, we show that links are, in fact, symmetric. In Section 4.2, we show that, through the use of TSCH, the low-power wireless topology is, in fact, extremely stable.

\subsection{Link (A)Symmetry}

Motes report the average RSSI value of the packets received from each neighbor in their HR_NEIGHBORS health reports. Because the network uses channel hopping, these reported RSSI values are also averaged over 15 IEEE802.15.4 frequencies [1]. In this section, we use the term "RSSI" to denote the average RSSI over 15 frequencies.

A common assumption is that links between neighbor low-power wireless devices are hugely asymmetric. That is, on a link between nodes $A$ and $B, A$ receives $B$ 's link-layer frames with an RSSI very different from the frames $B$ receives from $A$. Numerous routing protocols (often standardized [3]) reuse that assumption and start with a costly step of filtering out asymetric links.

We look at the link statistics between the 18th of June 2016 and the 4th of July 2016 (16 days). The sample contains 411,132 HR_NEIGHBORS messages received from 14 DC9003 nodes (same hardware). During that period, 21 links are active with at least 250 transmissions for each link. For each of those links, we compute the difference between average RSSI in each directions. Results are presented in Fig. 5.

Fig. 5 shows that the RSSI difference never exceeds a couple of dB. Looking at Fig. 4, this translates into a handful of percentage points difference in PDR only. This means the links can be considered symmetric. This result is in-line with the physical phenomenon that the signal tranveling from $A$ to $B$ undergoes the same attenuation as that from $B$ to $A$. This result would not hold if the neighbor radios had a different transmit power or sensitivity. That being said, discussions on link symmetry at the routing layer is largely artificial, as any "good" medium access control (MAC) protocol uses link-layer acknowledgments.

\subsection{Network Stability}

Wireless in unreliable in nature. It is normal that some wireless links interconnecting motes "come and go". That is, links that have been performing well (e.g. PDR ¿90\%) can suddenly disappear (e.g. PDR;10\%). Similarly, nodes that were not able to communicate can suddenly hear one another perfectly.

The question, however, is what time scale is considered. Early academic work on low-power wireless [6] has looked at the "burtiness" of the wireless links, i.e. changes over the course of 10-1000's ms. Some follow-up work has taken the assumption that wireless links are so unstable that only a reactive routing approach works. In this section, we infirm this statement by looking at the stability of the network. 


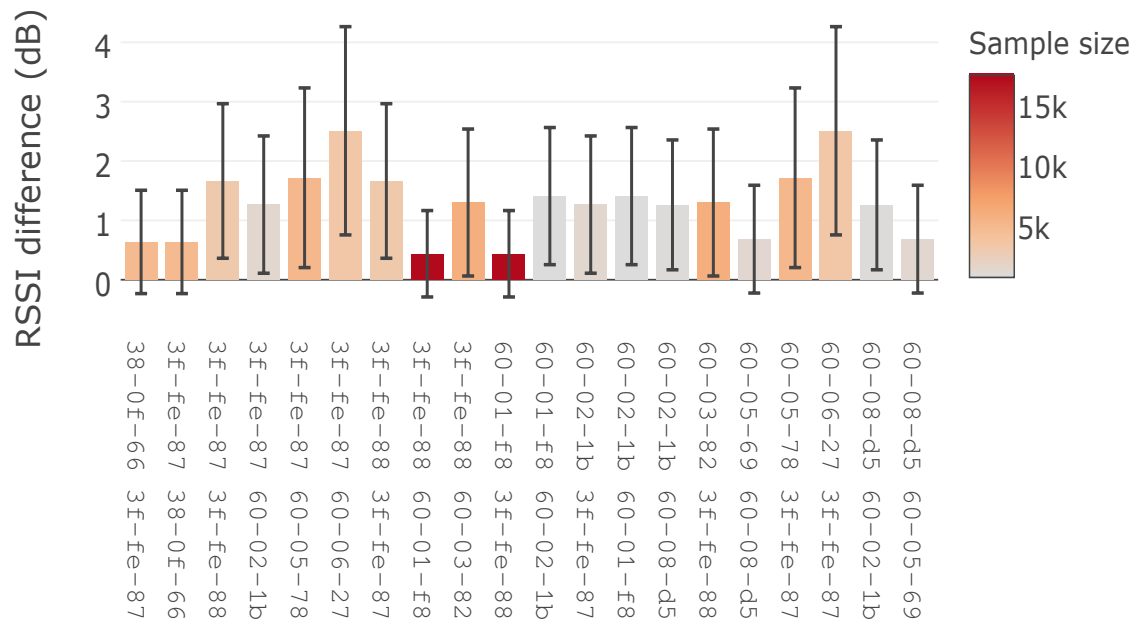

Figure 5: The difference in RSSI between the two directions of 20 wireless links (links continuously active in the 18-25 June 2016 period). The average value (the bar) is complemented with the standard deviation. The color of the bar indicates sample size.

In particular, we look at the path_delete and path_create events. These are generated each time a node adds/deletes a neighbor to communicate with, which happens for example when the routing topology changes (see Section 2). The number of path_delete and path_create events is a direct measurement of the network stability. Note that we remove node b0-00-cc from the dataset as it does not respect the Dust requirement of having at least two parents to associate with. Due to the lack of second parent, the node was producing over 20 times the amount of messages than all the other nodes assembled.

Fig. 6 shows the number of path_delete and path_create events per day, over a 16-day period. For reference, the total number of links in the network is also depicted. There are less than 5 path_delete or path_create events per day in the entire network. This means that links, once established, remain useful for days/weeks at a time, and that the network is extremely stable.

This stability can largely be attributed to the use of channel hopping. Changing frequency for each packet is known to efficiently combat multi-path fading and external interference [9], the major causes of instability. It does not contradict the findings of [6], it just means that link-layer retransmissions can efficiently cope with link burstiness, and that the multi-hop topology can remain very stable. 


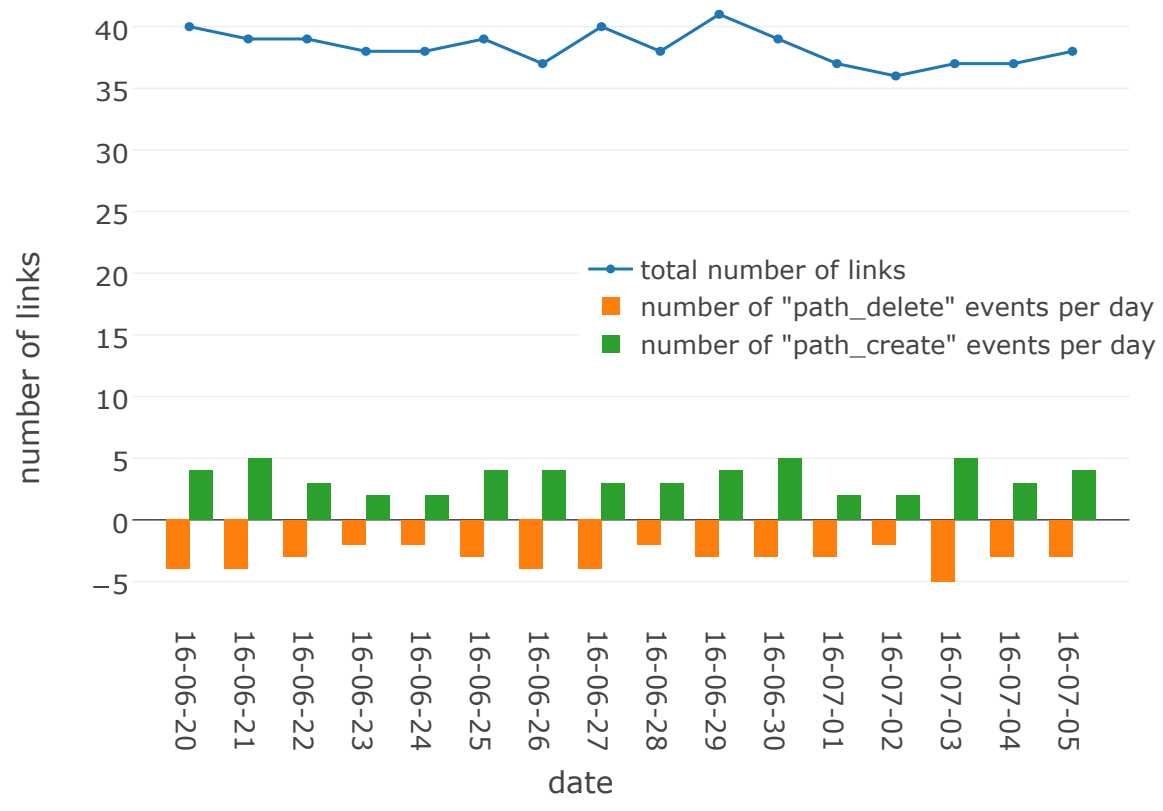

Figure 6: Network stability: the number of path_create and path_delete events generated per day over a 16-day period. The top portion shows the total number of links. 


\section{Conclusion}

This paper analyzes the 369,276 network statistics generated by a 21 -mote lowpower wireless mesh networks deployed in a peach orchard in Argentina, over the course of 3 months.

We use a "waterfall" plot to show that the network does not suffer from severe interferences from different wireless devices deployed in the same area. The SmartMesh IP network delivers its exceptional performance, with 0 packets lost out of 693,844 received (100\% reliable) and 4-16 years of battery lifetime on a pair of commercial AA batteries.

While it is often assumed that wireless links are asymmetric, we show to the contrary that the difference in RSSI averaged over 15 IEEE802.15.4 channels does not exceed a handful of $\mathrm{dB}$. We show that the network is extremely stable, with less than 5 links being added or deleted per day.

We attribute this performance to the use of Time Synchronized Channel Hopping (TSCH) technology at the heart of the SmartMesh IP products.

\section{References}

[1] 802.15.4-2011: IEEE Standard for Local and metropolitan area networks. Part 15.4: Low-Rate Wireless Personal Area Networks (LR-WPANs), 5 September 2011.

[2] 802.15.4e-2012: IEEE Standard for Local and metropolitan area networksPart 15.4: Low-Rate Wireless Personal Area Networks (LR-WPANs) Amendment 1: MAC sublayer, 16 April 2012.

[3] T. H. Clausen and P. Jacquet. Optimized Link State Routing Protocol (OLSR), October 2003.

[4] Linear Technology. SmartMesh IP Application Notes, 2015. 
[5] S. R. Saunders and A. Aragón-Zavala. Antennas and Propagation for Wireless Communication Systems. Wiley-Blackwell, 2nd edition, 2007.

[6] K. Srinivasan, M. A. Kazandjieva, S. Agarwal, and P. Levis. The $\beta$-factor: Measuring Wireless Link Burstiness. In Conference on Embedded Network Sensor Systems (SenSys), pages 29-42, Raleigh, NC, USA, 2008. ACM.

[7] T. Watteyne, A. L. Diedrichs, K. Brun-Laguna, J. E. Chaar, D. Dujovne, J. C. Taffernaberry, and G. Mercado. PEACH: Predicting Frost Events in Peach Orchards Using IoT Technology. EAI Endorsed Transactions on the Internet of Things, June 2016.

[8] T. Watteyne, S. Lanzisera, A. Mehta, and K. S. Pister. Mitigating Multipath Fading through Channel Hopping in Wireless Sensor Networks. In IEEE International Conference on Communications (ICC), pages 1-5, Cape Town, South Africa, 23-27 May 2010. IEEE.

[9] T. Watteyne, A. Mehta, and K. Pister. Reliability Through Frequency Diversity: Why Channel Hopping Makes Sense. In International Symposium on Performance Evaluation of Wireless Ad Hoc, Sensor, and Ubiquitous Networks (PE-WASUN), pages 116-123, Tenerife, Canary Islands, Spain, 26-30 October 2009. ACM.

[10] T. Watteyne, J. Weiss, L. Doherty, and J. Simon. Industrial IEEE802.15.4e Networks: Performance and Trade-offs. In International Conference on Communications (ICC), Internet of Things Symposium, London, UK, 8-12 June 2015. IEEE.

[11] S. Zats. Wireless Sensor Networks Scaling and Deployment in Industrial Automation. Master's thesis, University of California, Berkeley, 13 May 2010. 